\title{
Finite genus solutions to the lattice Schwarzian Korteweg-de Vries equation
}

\author{
Xiaoxue $\mathrm{Xu}^{\mathrm{a}}$, Cewen $\mathrm{Cao}^{\mathrm{a}}$, Guangyao Zhang ${ }^{\mathrm{b}}$ \\ ${ }^{a}$ School of Mathematics and Statistics, Zhengzhou University, Zhengzhou, 450001, PR \\ China \\ b School of Science, Huzhou University, Zhejiang, 313000, PR China \\ E-mail: xiaoxuexu@zzu.edu.cn
}

\begin{abstract}
Based on integrable Hamiltonian systems related to the derivative Schwarzian Korteweg-de Vries (SKdV) equation, a novel discrete Lax pair for the lattice $\mathrm{SKdV}(\mathrm{SKdV})$ equation is given by two copies of a Darboux transformation which can be used to derive an integrable symplectic correspondence. Resorting to the discrete version of Liouville-Arnold theorem, finite genus solutions to the $\mathrm{ISKdV}$ equation are calculated through Riemann surface method.
\end{abstract}

Keyword: lattice Schwarzian Korteweg-de Vries equation; integrable symplectic map; finite genus solution.

2000 Mathematics Subject Classification: 37J10, 37K10, 39A13

\section{Introduction}

Remarkable progress has been made in recent years in the study of discrete soliton equations (see 12 and the references therein). Among the related mathematical theories, the property of multi-dimensional consistency plays an important role in the understanding of discrete integrability. In the 2-dimensional case, it leads to the well-known AdlerBobenko-Suris (ABS) list [1, 10, which gives a classification of integrable quadrilateral lattice equations. Quite a few works have appeared in the study of the ABS equations, concerning their relations with the usual soliton equations, the Lax pairs, explicit analytic solutions, Bäcklund transformations (BTs), symmetries and conservation laws etc. $3,6,7,14,15,19,21,22,26,32,33$. 
The purpose of this paper is to investigate the ISKdV equation, which was first given in [23],

$$
\Xi:=\gamma_{1}^{2}(u-\hat{u})(\tilde{u}-\hat{\tilde{u}})-\gamma_{2}^{2}(u-\tilde{u})(\hat{u}-\hat{\tilde{u}})=0,
$$

where the usual notation is adopted: $u=u(m, n), \tilde{u}=u(m+1, n), \hat{u}=u(m, n+1)$. Eq. (1.1) is exactly the Q1(0) model (Q1 with $\delta=0)$ in the ABS hierarchy. The approach of Lax representation will be used to confirm the integrability of Eq. (1.1) and to calculate its basic explicit analytic solutions, the finite genus solutions [6, 7].

To produce a purely discrete Lax pair, it is vital to select two appropriate discrete spectral problems. It turns out that a special role is played by the semi-discrete integrable equations, which are also of independent interest, see [18] and references therein, where the well-known Toda, the Volterra and the Ablowitz-Ladik hierarchies are investigated thoroughly. A semi-discrete Lax pair can be constructed with the help of a continuous spectral problem and its Darboux transformation (DT), where the DT is regarded as a discrete spectral problem [4, 18, which usually leads to an integrable symplectic map by using the non-linearization technique [5] 7]. Refer to [16], integrable maps are called BTs whose geometrical explanation is given in terms of spectral curves and their Jacobians. And the symplectic correspondences (BTs) compatible with finite gap solutions of $\mathrm{KdV}$ have been discussed through DTs for the standard KdV spectral problem [13].

In our case we consider the continuous SKdV equation,

$$
\frac{\phi_{y}}{\phi_{x}}+\frac{1}{4} S[\phi ; x]=0
$$

where $S[\phi ; x]$ denotes the Schwarzian derivative of $\phi[20,30,31$, i.e.

$$
\begin{aligned}
S[\phi ; x] & =\left(\frac{\phi_{x x}}{\phi_{x}}\right)_{x}-\frac{1}{2}\left(\frac{\phi_{x x}}{\phi_{x}}\right)^{2}, \\
& =\frac{\phi_{x x x}}{\phi_{x}}-\frac{3}{2}\left(\frac{\phi_{x x}}{\phi_{x}}\right)^{2} .
\end{aligned}
$$

Technically, it is more convenient to use the derivative version

$$
w_{y}+\frac{1}{4}\left(w_{x x}-\frac{3 w_{x}^{2}}{2 w}\right)_{x}=0
$$

with $w=\phi_{x}$. The Eq. (1.4) has a Lax pair given by

$$
\begin{aligned}
& \partial_{x} \chi=\left(\begin{array}{cc}
0 & -w \lambda^{-1} \\
w^{-1} \lambda^{-1} & 0
\end{array}\right) \chi \\
& \partial_{y} \chi=\left(\begin{array}{cc}
-\frac{w_{x}}{2 w} \lambda^{-2} & -w \lambda^{-3}+\frac{1}{4}\left(w_{x x}-\frac{3 w_{x}^{2}}{2 w}\right) \lambda^{-1} \\
w^{-1} \lambda^{-3}+\frac{1}{4 w^{2}}\left(w_{x x}-\frac{w_{x}^{2}}{2 w}\right) \lambda^{-1} & \frac{w_{x}}{2 w} \lambda^{-2}
\end{array}\right) \chi .
\end{aligned}
$$


We note that in 24], the Lax pair for one Schwarzian PDE, which is equivalent to the SKdV hierarchy via expansions on the independent variables and has a fully discrete counterpart (1.1) by considering the independent variables as lattice parameters, has been found. However, we have not been able to blend it with the algebro-geometric technique of nonlinearization employed in the present paper. Fortunately, here each of the linear systems (1.5) and (1.6) can be nonlinearized to produce an integrable Hamiltonian system. Thus we find a Liouville integrable system associated with a spectral problem (see Sec. 2) given by

$$
\partial_{x} \chi=U(\lambda, u) \chi, \quad U(\lambda, u)=\left(\begin{array}{cc}
u & \lambda \\
0 & -u
\end{array}\right),
$$

and find that the following DT of Eq. (1.7) is critical:

$$
\tilde{\chi}=\left(\lambda^{2}-\gamma^{2}\right)^{-1 / 2} D^{(\gamma)}(\lambda, b) \chi, \quad D^{(\gamma)}(\lambda, b)=\left(\begin{array}{cc}
\lambda & \gamma b \\
\gamma b^{-1} & \lambda
\end{array}\right) .
$$

The compatibility condition $D_{x}^{(\gamma)}=\tilde{U} D^{(\gamma)}-D^{(\gamma)} U$ gives rise to

$$
b_{x} / b=u+\tilde{u}, \quad \gamma b^{-1}=u-\tilde{u} .
$$

This suggests a constraint $b=\gamma /(u-\tilde{u})$ and leads to a Lax pair, different from the one in [23], for Eq. (1.1).

Lemma 1.1 The lSKdV equation (1.1) has a Lax pair

$$
\begin{aligned}
& \tilde{\chi}=\left(\lambda^{2}-\gamma_{1}^{2}\right)^{-1 / 2} D^{\left(\gamma_{1}\right)}\left(\lambda, b^{\prime}\right) \chi, \quad b^{\prime}=\gamma_{1} /(u-\tilde{u}), \\
& \hat{\chi}=\left(\lambda^{2}-\gamma_{2}^{2}\right)^{-1 / 2} D^{\left(\gamma_{2}\right)}\left(\lambda, b^{\prime \prime}\right) \chi, \quad b^{\prime \prime}=\gamma_{2} /(u-\hat{u}),
\end{aligned}
$$

with

$$
\hat{D}^{\left(\gamma_{1}\right)} D^{\left(\gamma_{2}\right)}-\tilde{D}^{\left(\gamma_{2}\right)} D^{\left(\gamma_{1}\right)}=\frac{1}{\Upsilon}\left(\begin{array}{cc}
(u-\tilde{u})(u-\hat{u}) & \lambda(\hat{\tilde{u}}-\tilde{u}-\hat{u}+u) \\
0 & -(\tilde{u}-\hat{\tilde{u}})(\hat{u}-\hat{\tilde{u}})
\end{array}\right) \Xi,
$$

where $\Upsilon=(u-\tilde{u})(u-\hat{u})(\tilde{u}-\hat{\tilde{u}})(\hat{u}-\hat{\tilde{u}})$ and $\Xi$ is defined by Eq. (1.1).

The paper is organised as follows. In Sec. 2, a finite-dimensional Hamiltonian system which is a nonlinear version of the spectral problem (1.7) is presented. In Sec. 3, resorting to the Hamiltonian system, we construct an integrable symplectic map. In addition, with the help of the Burchnall-Chaundy theory, the discrete potential is expressed in terms of theta functions. In Sec. 4, based on the discrete version of the Liouville-Arnold theorem, the finite genus solutions of ISKdV equation (1.1) are obtained through the commutativity of integrable maps [7]. 


\section{The integrable Hamiltonian system $\left(H_{1}\right)$}

Take the symplectic manifold $\left(\mathbb{R}^{2 N}, \mathrm{~d} p \wedge \mathrm{d} q\right)$ as the phase space. The symplectic coordinate is defined as $(p, q)=\left(p_{1}, \ldots, p_{N}, q_{1}, \ldots, q_{N}\right)$. Let $A=\operatorname{diag}\left(\alpha_{1}, \cdots, \alpha_{N}\right)$ with distinct, non-zero $\alpha_{1}^{2}, \cdots, \alpha_{N}^{2}$. Define a Lax matrix

$$
L(\lambda ; p, q)=\sigma_{+}+\frac{1}{2} \sum_{j=1}^{N}\left(\frac{\varepsilon_{j}}{\lambda-\alpha_{j}}+\frac{\sigma_{3} \varepsilon_{j} \sigma_{3}}{\lambda+\alpha_{j}}\right)=\left(\begin{array}{cc}
\lambda Q_{\lambda}(p, q) & 1-Q_{\lambda}(A p, p) \\
Q_{\lambda}(A q, q) & -\lambda Q_{\lambda}(p, q)
\end{array}\right)
$$

where $\sigma_{+}, \sigma_{3}$ are the usual Pauli matrices, and

$$
\varepsilon_{j}=\left(\begin{array}{cc}
p_{j} q_{j} & -p_{j}^{2} \\
q_{j}^{2} & -p_{j} q_{j}
\end{array}\right), \quad Q_{\lambda}(\xi, \eta)=\sum_{j=1}^{N} \frac{\xi_{j} \eta_{j}}{\lambda^{2}-\alpha_{j}^{2}}
$$

$\forall(\xi, \eta)=\left(\xi_{1}, \ldots, \xi_{N}, \eta_{1}, \ldots, \eta_{N}\right) \in \mathbb{R}^{2 N}$.

The generating function $\mathcal{F}_{\lambda}=\operatorname{det} L(\lambda ; p, q)$ is a rational function of the argument $\zeta=\lambda^{2}$,

$$
\mathcal{F}_{\lambda}(p, q)=\left(Q_{\lambda}(A p, p)-1\right) Q_{\lambda}(A q, q)-\lambda^{2} Q_{\lambda}^{2}(p, q)
$$

The expansion $\mathcal{F}_{\lambda}=\sum_{l=1}^{\infty} F_{l} \zeta^{-l}$ gives rise to a set of quantities on phase space as

$$
\begin{gathered}
F_{1}=-<A q, q>-<p, q>^{2}, \\
F_{l}=-<A^{2 l-1} q, q>+\sum_{j+k=l ; j, k \geq 1}<A^{2 j-1} p, p><A^{2 k-1} q, q> \\
-\sum_{j+k=l+1 ; j, k \geq 1}<A^{2 j-2} p, q><A^{2 k-2} p, q>,
\end{gathered}
$$

$(l=1,2, \cdots)$, where $<\xi, \eta>=\Sigma_{j=1}^{N} \xi_{j} \eta_{j}$. Consider the Hamiltonian system $\left(H_{1}\right)$, defined by the Hamiltonian function

$$
\begin{aligned}
& H_{1}=\frac{F_{1}}{2}=-\frac{1}{2}<A q, q>-\frac{1}{2}<p, q>^{2}, \\
& \partial_{x}\left(\begin{array}{c}
p_{j} \\
q_{j}
\end{array}\right)=\left(\begin{array}{c}
-\partial H_{1} / \partial q_{j} \\
\partial H_{1} / \partial p_{j}
\end{array}\right)=\left(\begin{array}{cc}
<p, q> & \alpha_{j} \\
0 & -<p, q>
\end{array}\right)\left(\begin{array}{c}
p_{j} \\
q_{j}
\end{array}\right), \quad 1 \leq j \leq N .
\end{aligned}
$$

They are exactly $N$ copies of Eq. (1.7) with distinct $\lambda=\alpha_{j}$ and the constraint

$$
u=f_{U}(p, q)=<p, q>
$$

In this context $\left(H_{1}\right)$ is called a non-linearization of the linear spectral problem (1.7).

According to the Liouville-Arnold theory [2], we shall discuss the coefficients $F_{1}, \cdots, F_{N}$ given by (2.3) are first integrals of the phase flow with Hamiltonian function $H_{1}$, i.e., 
$\left\{F_{j}, H_{1}\right\}=0,(j=1, \ldots, N)$, where $\{\cdot, \cdot\}$ denotes the Poisson bracket on the phase space. The involution and functional independence between $F_{1}, \cdots, F_{N}$ guarantee that the Hamiltonian system $\left(H_{1}\right)$ is completely integrable.

Consider the Hamiltonian system $\left(\mathcal{F}_{\lambda}\right)$,

$$
\begin{aligned}
\frac{\mathrm{d}}{\mathrm{d} t_{\lambda}}\left(\begin{array}{c}
p_{j} \\
q_{j}
\end{array}\right) & =\left(\begin{array}{c}
-\partial \mathcal{F}_{\lambda} / \partial q_{j} \\
\partial \mathcal{F}_{\lambda} / \partial p_{j}
\end{array}\right)=W\left(\lambda, \alpha_{j}\right)\left(\begin{array}{c}
p_{j} \\
q_{j}
\end{array}\right), \\
W(\lambda, \mu) & =\frac{2}{\lambda^{2}-\mu^{2}}\left(\begin{array}{cc}
\lambda L^{11}(\lambda) & \mu L^{12}(\lambda) \\
\mu L^{21}(\lambda) & -\lambda L^{11}(\lambda)
\end{array}\right)=\frac{L(\lambda)}{\lambda-\mu}+\frac{\sigma_{3} L(\lambda) \sigma_{3}}{\lambda+\mu},
\end{aligned}
$$

where $L(\lambda)$ is the abbreviation of $L(\lambda ; p, q)$ and $L^{i j}(\lambda), i, j=1,2$ are entries of the matrix $L(\lambda)$. Hence we obtain $\mathrm{d} \varepsilon_{j} / \mathrm{d} t_{\lambda}=\left[W\left(\lambda, \alpha_{j}\right), \varepsilon_{j}\right]$, where $[\cdot, \cdot]$ stands for the matrix commutator. Based on this formula, it is easy to derive the following basic equation,

$$
\frac{\mathrm{d}}{\mathrm{d} t_{\lambda}} L(\mu)=[W(\lambda, \mu), L(\mu)], \quad \forall \lambda, \mu \in \mathbb{C} .
$$

As a corollary, we have

$$
\begin{aligned}
& \left\{\mathcal{F}_{\mu}, \mathcal{F}_{\lambda}\right\}=0, \quad \forall \lambda, \mu \in \mathbb{C} ; \\
& \left\{F_{j}, F_{k}\right\}=0, \quad j, k=1,2, \cdots .
\end{aligned}
$$

Actually, by equation (2.7), $\left(\mathrm{d} / \mathrm{d} t_{\lambda}\right) L^{2}(\mu)=\left[W(\lambda, \mu), L^{2}(\mu)\right]$. Since $L^{2}(\mu)=-I \mathcal{F}_{\mu}$, where $I$ is the identity matrix, we have $\mathrm{d} \mathcal{F}_{\mu} / \mathrm{d} t_{\lambda}=0$. According to the definition of Poisson bracket [2, this is exactly Eq. (2.8), whose power series expansion gives rise to Eq. (2.9).

The generating function $\mathcal{F}_{\lambda}$ has a factorization

$$
\mathcal{F}_{\lambda}=F_{1} \frac{Z(\zeta)}{\alpha(\zeta)}=F_{1} \frac{R(\zeta)}{\zeta \alpha^{2}(\zeta)}
$$

with $\alpha(\zeta)=\Pi_{j=1}^{N}\left(\zeta-\alpha_{j}^{2}\right), Z(\zeta)=\Pi_{k=1}^{N-1}\left(\zeta-\zeta_{k}\right), R(\zeta)=\zeta \alpha(\zeta) Z(\zeta)$, where $F_{1}$ is given by Eq. (2.3). The spectral curve is defined as

$$
\mathcal{R}: \xi^{2}-R(\zeta)=0
$$

which is hyperelliptic with genus $g=N-1$ and has two points at infinity, $\infty_{+}, \infty_{-}$. At the branch point $\mathfrak{o}=(\zeta=0, \xi=0), \mathcal{R}$ has a local coordinate $\lambda=\zeta^{1 / 2}$. The generic point on $\mathcal{R}$ is given as

$$
\mathfrak{p}(\zeta)=(\zeta, \xi=\sqrt{R(\zeta)}), \quad(\tau \mathfrak{p})(\zeta)=(\zeta, \xi=-\sqrt{R(\zeta)})
$$


where $\tau: \mathcal{R} \rightarrow \mathcal{R}$ is the hyperelliptic involution. The variables $\left\{\nu_{j}^{2}\right\}$ defined as the roots of the equation

$$
L^{21}(\lambda)=\sum_{j=1}^{N} \frac{\alpha_{j} q_{j}^{2}}{\lambda^{2}-\alpha_{j}^{2}}=<A q, q>\frac{\mathfrak{n}(\zeta)}{\alpha(\zeta)}=0, \quad \mathfrak{n}(\zeta)=\prod_{j=1}^{g}\left(\zeta-\nu_{j}^{2}\right),
$$

give an elliptic coordinate system [17. By Eq. (2.7) we have

$$
\frac{\mathrm{d}}{\mathrm{d} t_{\lambda}} L^{21}(\mu)=2\left(W^{21}(\lambda, \mu) L^{11}(\mu)-W^{11}(\lambda, \mu) L^{21}(\mu)\right) .
$$

Putting $\mu=\nu_{k}$, with $L^{11}\left(\nu_{k}\right)=\sqrt{-F_{1} \cdot R\left(\nu_{k}^{2}\right)} /\left(\nu_{k} \alpha\left(\nu_{k}^{2}\right)\right)$ from Eq. (2.10), we get the evolution of the elliptic variables along the $\mathcal{F}_{\lambda}$-flow,

$$
\begin{aligned}
& \frac{1}{2 \sqrt{R\left(\nu_{k}^{2}\right)}} \cdot \frac{\mathrm{d}\left(\nu_{k}^{2}\right)}{\mathrm{d} t_{\lambda}}=-\frac{2 \sqrt{-F_{1}}}{\alpha(\zeta)} \cdot \frac{\mathfrak{n}(\zeta)}{\left(\zeta-\nu_{k}^{2}\right) \mathfrak{n}^{\prime}\left(\nu_{k}^{2}\right)}, \quad 1 \leq k \leq g, \\
& \sum_{k=1}^{g} \frac{\left(\nu_{k}^{2}\right)^{g-s}}{2 \sqrt{R\left(\nu_{k}^{2}\right)}} \cdot \frac{\mathrm{d}\left(\nu_{k}^{2}\right)}{\mathrm{d} t_{\lambda}}=-\frac{2 \sqrt{-F_{1}}}{\alpha(\zeta)} \cdot \zeta^{g-s}, \quad 1 \leq s \leq g,
\end{aligned}
$$

where the interpolation formula of polynomials is used. With the help of the quasi-AbelJacobi variables

$$
\phi_{s}^{\prime}=\sum_{k=1}^{g} \int_{\mathfrak{p}_{0}}^{\mathfrak{p}\left(\nu_{k}^{2}\right)} \omega_{s}^{\prime}, \quad \omega_{s}^{\prime}=\frac{\zeta^{g-s} \mathrm{~d} \zeta}{2 \sqrt{R(\zeta)}}, \quad 1 \leq s \leq g,
$$

Eq. (2.15) is rewritten in a simple form and gives rise to

Proposition 2.1 The $\mathcal{F}_{\lambda}$ - and the $F_{l}$-flow are linearized by $\phi_{s}^{\prime}$ as

$$
\begin{aligned}
& \frac{\mathrm{d} \phi_{s}^{\prime}}{\mathrm{d} t_{\lambda}}=\left\{\phi_{s}^{\prime}, \mathcal{F}_{\lambda}\right\}=-\frac{2 \sqrt{-F_{1}}}{\alpha(\zeta)} \cdot \zeta^{g-s}, \quad 1 \leq s \leq g, \\
& \frac{\mathrm{d} \phi_{s}^{\prime}}{\mathrm{d} t_{l}}=\left\{\phi_{s}^{\prime}, F_{l}\right\}=-2 \sqrt{-F_{1}} \cdot A_{l-s-1}, \quad l=1,2, \cdots,
\end{aligned}
$$

where $A_{0}=1 ; A_{-j}=0,(j=1,2, \cdots)$; while $A_{j},(j=1,2, \cdots)$, are defined by

$$
\frac{\zeta^{N}}{\alpha(\zeta)}=\frac{1}{\Pi_{k=1}^{N}\left(1-\alpha_{k}^{2} \zeta^{-1}\right)}=\sum_{j=0}^{\infty} A_{j} \zeta^{-j}
$$

In particular, $\left\{\phi_{s}^{\prime}, F_{1}\right\}=0,1 \leq s \leq g$.

Proposition 2.2 The Hamiltonian system $\left(H_{1}\right)$ is integrable, possessing $N$ integrals $F_{1}, \cdots, F_{N}$, involutive with each other and functionally independent in the dense, open subset $\mathcal{O}=$ $\left\{(p, q) \in \mathbb{R}^{2 N}: F_{1} \neq 0\right\}$. 
Proof. $F_{l}$ is an integral since $\left\{H_{1}, F_{l}\right\}=(1 / 2)\left\{F_{1}, F_{l}\right\}=0$ by Eq. (2.9). It needs only to prove that $\mathrm{d} F_{1}, \cdots, \mathrm{d} F_{N}$ are linearly independent in $T_{(p, q)}^{*} \mathbb{R}^{2 N}$ at $(p, q) \in \mathcal{O}$. Suppose $\sum_{j=1}^{N} c_{j} \mathrm{~d} F_{j}=0$. Then

$$
c_{2}\left\{\phi_{s}^{\prime}, F_{2}\right\}+\cdots+c_{N}\left\{\phi_{s}^{\prime}, F_{N}\right\}=0, \quad 1 \leq s \leq N-1
$$

By Eq. (2.18), the coefficient matrix is non-degenerate,

$$
\left(\begin{array}{ccc}
\left\{\phi_{1}^{\prime}, F_{2}\right\} & \cdots & \left\{\phi_{1}^{\prime}, F_{N}\right\} \\
\vdots & \ddots & \vdots \\
\left\{\phi_{g}^{\prime}, F_{2}\right\} & \cdots & \left\{\phi_{g}^{\prime}, F_{N}\right\}
\end{array}\right)=-2 \sqrt{-F_{1}} \cdot\left(\begin{array}{ccccc}
1 & A_{1} & A_{2} & \cdots & A_{g-1} \\
& 1 & A_{1} & \cdots & A_{g-2} \\
& & \ddots & \cdots & \vdots \\
& & & 1 & A_{1} \\
& & & & 1
\end{array}\right)
$$

Thus $c_{2}=\cdots=c_{N}=0$ and $c_{1} \mathrm{~d} F_{1}=0$. We have $c_{1}=0$ since $\mathrm{d} F_{1} \neq 0$ at $\mathcal{O}$. Otherwise,

$$
-\frac{1}{2} \mathrm{~d} F_{1}=\sum_{j=1}^{N}\left(<p, q>q_{j} \mathrm{~d} p_{j}+\left(\alpha_{j} q_{j}+<p, q>p_{j}\right) \mathrm{d} q_{j}\right)=0 .
$$

Hence $\alpha_{j} q_{j}+<p, q>p_{j}=0, \forall j$; and $F_{1}=0$. This is a contradiction.

\section{$3 \quad$ The integrable symplectic map $\mathcal{S}_{\gamma}$}

As a non-linearization of Eq. (1.8) , define a map $\mathcal{S}_{\gamma}: \mathbb{R}^{2 N} \rightarrow \mathbb{R}^{2 N},(p, q) \mapsto(\tilde{p}, \tilde{q})$ by

$$
\left(\begin{array}{c}
\tilde{p}_{j} \\
\tilde{q}_{j}
\end{array}\right)=\left(\alpha_{j}^{2}-\gamma^{2}\right)^{-1 / 2} D^{(\gamma)}\left(\alpha_{j}, b\right)\left(\begin{array}{c}
p_{j} \\
q_{j}
\end{array}\right), \quad 1 \leq j \leq N
$$

where a constraint $b=f_{\gamma}(p, q)$ is to be chosen so that $\mathcal{S}_{\gamma}$ is integrable and symplectic.

Lemma 3.1 Let $P^{(\gamma)}(b ; p, q)=b^{2} L^{21}(\gamma)+2 b L^{11}(\gamma)-L^{12}(\gamma)$. Then

$$
\begin{aligned}
& L(\lambda ; \tilde{p}, \tilde{q}) D^{(\gamma)}(\lambda, b)-D^{(\gamma)}(\lambda, b) L(\lambda ; p, q)=-\gamma b^{-1} P^{(\gamma)}(b ; p, q) \sigma_{3}, \\
& \sum_{j=1}^{N}\left(\mathrm{~d} \tilde{p}_{j} \wedge \mathrm{d} \tilde{q}_{j}-\mathrm{d} p_{j} \wedge \mathrm{d} q_{j}\right)=\frac{1}{2} \gamma b^{-2} \mathrm{~d} P^{(\gamma)}(b ; p, q) \wedge \mathrm{d} b .
\end{aligned}
$$

Proof. By Eq. (3.1), we get $\tilde{\varepsilon}_{j} D^{(\gamma)}\left(\alpha_{j}\right)-D^{(\gamma)}\left(\alpha_{j}\right) \varepsilon_{j}=0$. Besides, we have $\sigma_{3}^{2}=I$ and

$$
\begin{aligned}
& \sigma_{3} D^{(\gamma)}(\lambda) \sigma_{3}=-D^{(\gamma)}(-\lambda), \\
& D^{(\gamma)}( \pm \lambda)-D^{(\gamma)}\left(\alpha_{j}\right)= \pm\left(\lambda \mp \alpha_{j}\right) I .
\end{aligned}
$$


Based on these preparations, we calculate the left-hand side of Eq. (3.2),

$$
\begin{aligned}
{\left[\sigma_{+}, D^{(\gamma)}(\lambda)\right] } & +\frac{1}{2} \sum_{j=1}^{N}\left(\frac{\tilde{\varepsilon}_{j} D^{(\gamma)}(\lambda)-D^{(\gamma)}(\lambda) \varepsilon_{j}}{\lambda-\alpha_{j}}+\frac{\sigma_{3} \tilde{\varepsilon}_{j} \sigma_{3} D^{(\gamma)}(\lambda)-D^{(\gamma)}(\lambda) \sigma_{3} \varepsilon_{j} \sigma_{3}}{\lambda+\alpha_{j}}\right) \\
& =\gamma b^{-1} \sigma_{3}+\frac{1}{2} \sum_{j=1}^{N}\left(\left(\tilde{\varepsilon}_{j}-\varepsilon_{j}\right)+\sigma_{3}\left(\tilde{\varepsilon}_{j}-\varepsilon_{j}\right) \sigma_{3}\right) \\
& =\left(\gamma b^{-1}+<\tilde{p}, \tilde{q}>-<p, q>\right) \sigma_{3} .
\end{aligned}
$$

By using Eq. (3.1), we obtain

$$
\gamma b^{-1}+<\tilde{p}, \tilde{q}>-<p, q>=-\gamma b^{-1} P^{(\gamma)}(b ; p, q) .
$$

This proves Eq. (3.2). Eq. (3.3) is obtained through direct calculations.

Consider the quadratic equation $P^{(\gamma)}(b)=0$, whose roots give the constraint on $b$,

$$
b=f_{\gamma}(p, q)=\frac{1}{Q_{\gamma}(A q, q)}\left(-\gamma Q_{\gamma}(p, q) \pm \sqrt{-\mathcal{F}_{\gamma}(p, q)}\right) .
$$

Actually $\gamma b$ can be written as a meromorphic function on $\mathcal{R}$,

$$
\mathfrak{b}(\mathfrak{p})=\frac{1}{Q_{\gamma}(A q, q)}\left(-\gamma^{2} Q_{\gamma}(p, q)+\sqrt{-F_{1}} \frac{\xi}{\alpha(\gamma)}\right) .
$$

Though doubled-valued as a function of $\beta \in \mathbb{C}$, it is single-valued as a function of $\mathfrak{p}\left(\beta^{2}\right) \in$ $\mathcal{R}$. Hence we obtain

Proposition 3.2 The map $\mathcal{S}_{\gamma}: \mathbb{R}^{2 N} \rightarrow \mathbb{R}^{2 N},(p, q) \mapsto(\tilde{p}, \tilde{q})$, defined as

$$
\left(\begin{array}{c}
\tilde{p}_{j} \\
\tilde{q}_{j}
\end{array}\right)=\left.\left(\alpha_{j}^{2}-\gamma^{2}\right)^{-1 / 2}\left(\begin{array}{c}
\alpha_{j} p_{j}+\gamma b q_{j} \\
\gamma b^{-1} p_{j}+\alpha_{j} q_{j}
\end{array}\right)\right|_{b=f_{\gamma}(p, q)}, \quad 1 \leq j \leq N,
$$

is symplectic and integrable, possessing the Liouville set of integrals

$$
F_{l}(\tilde{p}, \tilde{q})=F_{l}(p, q), \quad 1 \leq j \leq N
$$

Proof. Since $P^{(\gamma)}(b)=0$, by Eq. (3.2) and (3.3) we have

$$
\begin{aligned}
& L(\lambda ; \tilde{p}, \tilde{q}) D^{(\gamma)}\left(\lambda, f_{\gamma}(p, q)\right)-D^{(\gamma)}\left(\lambda, f_{\gamma}(p, q)\right) L(\lambda ; p, q)=0 \\
& \sum_{j=1}^{N} \mathrm{~d} \tilde{p}_{j} \wedge \mathrm{d} \tilde{q}_{j}=\sum_{j=1}^{N} \mathrm{~d} p_{j} \wedge \mathrm{d} q_{j} .
\end{aligned}
$$

Taking the determinant of Eq. (3.8), we obtain $\mathcal{F}_{\lambda}(\tilde{p}, \tilde{q})=\mathcal{F}_{\lambda}(p, q)$, hence Eq. (3.7).

By Eq. (3.7), the discrete flow $(p(m), q(m))=\mathcal{S}_{\gamma}^{m}\left(p_{0}, q_{0}\right)$ has constants of motion $\left\{F_{l}\right\}$. Define finite genus potentials as

$$
\begin{aligned}
& b(m)=b_{m}=f_{\gamma}(p(m), q(m)), \\
& u(m)=u_{m}=f_{U}(p(m), q(m))=<p(m), q(m)>.
\end{aligned}
$$


By Eq. (3.4), they have the relation

$$
b_{m}=\gamma /\left(u_{m}-u_{m+1}\right)
$$

which meets the requirement of Eq. (1.10). Along the $m$-flow, Eq. (3.8) is rewritten as

$$
L_{m+1}(\lambda) D_{m}^{(\gamma)}(\lambda)=D_{m}^{(\gamma)}(\lambda) L_{m}(\lambda)
$$

where $L_{m}(\lambda)=L(\lambda ; p(m), q(m)), D_{m}^{(\gamma)}(\lambda)=D^{(\gamma)}\left(\lambda, b_{m}\right)$. Now we calculate $u_{m}$ with the help of the following spectral problem and its fundamental solution matrix $M_{\gamma}(m, \lambda)$,

$$
\begin{aligned}
& h_{\gamma}(m+1, \lambda)=D_{m}^{(\gamma)}(\lambda) h_{\gamma}(m, \lambda) ; \\
& M_{\gamma}(m+1, \lambda)=D_{m}^{(\gamma)}(\lambda) M_{\gamma}(m, \lambda), \quad M_{\gamma}(0, \lambda)=I .
\end{aligned}
$$

By induction we have

$$
\begin{aligned}
& M_{\gamma}(m, \lambda)=D_{m-1}^{(\gamma)}(\lambda) D_{m-2}^{(\gamma)}(\lambda) \cdots D_{0}^{(\gamma)}(\lambda), \\
& \operatorname{det} M_{\gamma}(m, \lambda)=\left(\lambda^{2}-\gamma^{2}\right)^{m} \\
& L_{m}(\lambda) M_{\gamma}(m, \lambda)=M_{\gamma}(m, \lambda) L_{0}(\lambda) .
\end{aligned}
$$

Lemma 3.3 The following functions are polynomials of the argument $\zeta=\lambda^{2}$,

$$
\begin{aligned}
& M_{\gamma}^{11}(2 k, \lambda), \lambda^{-1} M_{\gamma}^{12}(2 k, \lambda), \lambda^{-1} M_{\gamma}^{21}(2 k, \lambda), M_{\gamma}^{22}(2 k, \lambda), \\
& \lambda^{-1} M_{\gamma}^{11}(2 k+1, \lambda), M_{\gamma}^{12}(2 k+1, \lambda), M_{\gamma}^{21}(2 k+1, \lambda), \lambda^{-1} M_{\gamma}^{22}(2 k+1, \lambda) .
\end{aligned}
$$

Besides, as $\lambda \rightarrow \infty$,

$$
M_{\gamma}(m, \lambda)=\left(\begin{array}{cc}
\lambda^{m}\left[1+O\left(\lambda^{-2}\right)\right] & O\left(\lambda^{m-1}\right) \\
O\left(\lambda^{m-1}\right) & \lambda^{m}\left[1+O\left(\lambda^{-2}\right)\right]
\end{array}\right) .
$$

By Eq. (3.13), the solution space $\mathcal{E}_{\lambda}$ of Eq. (3.14) is invariant under the action of the linear operator $L_{m}(\lambda)$, which has two eigenvalues $\rho_{\lambda}^{ \pm}= \pm \rho_{\lambda}$,

$$
\rho_{\lambda}=\sqrt{-\mathcal{F}_{\lambda}}=\sqrt{-F_{1}} \cdot \frac{\sqrt{R(\zeta)}}{\lambda \alpha(\zeta)} .
$$

They define a meromorphic function $\mathfrak{r}(\mathfrak{p})=\sqrt{-F_{1}} \xi(\mathfrak{p}) / \alpha(\zeta(\mathfrak{p}))$ on $\mathcal{R}$ with $\mathfrak{r}\left(\mathfrak{p}\left(\lambda^{2}\right)\right)=\lambda \rho_{\lambda}^{+}$, $\mathfrak{r}\left((\tau \mathfrak{p})\left(\lambda^{2}\right)\right)=\lambda \rho_{\lambda}^{-}$. The corresponding eigenvectors satisfy

$$
\begin{aligned}
& h_{ \pm}(m, \lambda)=\left(\begin{array}{c}
h_{ \pm}^{(1)}(m, \lambda) \\
h_{ \pm}^{(2)}(m, \lambda)
\end{array}\right)=M_{\gamma}(m, \lambda)\left(\begin{array}{c}
c_{\lambda}^{ \pm} \\
1
\end{array}\right), \\
& \left(L_{m}(\lambda)-\rho_{\lambda}^{ \pm}\right) h_{ \pm}(m, \lambda)=0 .
\end{aligned}
$$


Putting $m=0$, we solve

$$
\begin{aligned}
& c_{\lambda}^{ \pm}=\frac{L_{0}^{11}(\lambda) \pm \rho_{\lambda}}{L_{0}^{21}(\lambda)}=-\frac{L_{0}^{12}(\lambda)}{L_{0}^{11}(\lambda) \mp \rho_{\lambda}} \\
& c_{\lambda}^{+} c_{\lambda}^{-}=-\frac{L_{0}^{12}(\lambda)}{L_{0}^{21}(\lambda)}
\end{aligned}
$$

defining a meromorphic function $\mathfrak{c}(\mathfrak{p})$ with $\mathfrak{c}\left(\mathfrak{p}\left(\lambda^{2}\right)\right)=\lambda c_{\lambda}^{+}, \mathfrak{c}\left((\tau \mathfrak{p})\left(\lambda^{2}\right)\right)=\lambda c_{\lambda}^{-}$. As $\lambda \rightarrow \infty$,

$$
c_{\lambda}^{ \pm}=\left.\frac{<p, q> \pm \sqrt{-F_{1}}}{<A q, q>}\right|_{<p_{0}, q_{0}>} \lambda\left[1+O\left(\lambda^{-2}\right)\right] .
$$

Lemma 3.4 (Formula of Dubrovin-Novikov type.)

$$
\begin{aligned}
& \left.\left(\begin{array}{cc}
h_{+}^{(1)} h_{-}^{(1)} & h_{+}^{(1)} h_{-}^{(2)} \\
h_{+}^{(2)} h_{-}^{(1)} & h_{+}^{(2)} h_{-}^{(2)}
\end{array}\right)\right|_{(m, \lambda)}=\frac{\left(\lambda^{2}-\gamma^{2}\right)^{m}}{L_{0}^{21}(\lambda)}\left(\begin{array}{cc}
-L_{m}^{12}(\lambda) & L_{m}^{11}(\lambda)+\rho_{\lambda} \\
L_{m}^{11}(\lambda)-\rho_{\lambda} & L_{m}^{21}(\lambda)
\end{array}\right), \\
& h_{+}^{(2)}(m, \lambda) h_{-}^{(2)}(m, \lambda)=\frac{<A q, q>_{m}}{<A q, q>_{0}}\left(\zeta-\gamma^{2}\right)^{m} \prod_{j=1}^{g} \frac{\zeta-\nu_{j}^{2}(m)}{\zeta-\nu_{j}^{2}(0)} .
\end{aligned}
$$

Proof. Using Eq. (3.16), we calculate the left-hand side of Eq. (3.25),

$$
\begin{aligned}
L H S & =M_{\gamma}(m, \lambda)\left(\begin{array}{cc}
c_{\lambda}^{+} c_{\lambda}^{-} & c_{\lambda}^{+} \\
c_{\lambda}^{-} & 1
\end{array}\right) M_{\gamma}^{T}(m, \lambda) \\
& =\frac{1}{L_{0}^{21}(\lambda)} M_{\gamma}(m, \lambda)\left[L_{0}(\lambda)+\rho_{\lambda} I\right]\left(\begin{array}{cc}
0 & 1 \\
-1 & 0
\end{array}\right) M_{\gamma}^{T}(m, \lambda) \\
& =\frac{1}{L_{0}^{21}(\lambda)}\left[L_{m}(\lambda)+\rho_{\lambda} I\right] M_{\gamma}(m, \lambda)\left(\begin{array}{cc}
0 & 1 \\
-1 & 0
\end{array}\right) M_{\gamma}^{T}(m, \lambda) \\
& =\frac{1}{L_{0}^{21}(\lambda)}\left[L_{m}(\lambda)+\rho_{\lambda} I\right]\left(\begin{array}{cc}
0 & 1 \\
-1 & 0
\end{array}\right) \operatorname{det} M_{\gamma}(m, \lambda)=R H S .
\end{aligned}
$$

With the help of Eq. (2.12) and (3.25), Eq. (3.26) is verified by some calculations.

Lemma 3.5 As $\lambda \rightarrow \infty$,

$$
\begin{aligned}
& h_{ \pm}^{(1)}(m, \lambda)=\frac{<p, q>_{0} \pm \sqrt{-F_{1}}}{<A q, q>_{0}} \lambda^{m+1}\left[1+O\left(\lambda^{-2}\right)\right], \\
& h_{ \pm}^{(2)}(m, \lambda)=\frac{<p, q>_{m} \mp \sqrt{-F_{1}}}{<p, q>_{0} \mp \sqrt{-F_{1}}} \lambda^{m}\left[1+O\left(\lambda^{-2}\right)\right] .
\end{aligned}
$$

Proof. Since $h_{ \pm}^{(1)}(m, \lambda)=M_{\gamma}^{11}(m, \lambda) c_{\lambda}^{ \pm}+M_{\gamma}^{12}(m, \lambda)$, we have Eq. (3.27) in virtue of Eq. (3.18) and (3.24). By Eq. (3.25) we get

$$
\begin{aligned}
& \left.h_{+}^{(1)} h_{-}^{(2)}\right|_{(m, \lambda)}=\left(\lambda^{2}-\gamma^{2}\right)^{m} \frac{L_{m}^{11}(\lambda)+\rho_{\lambda}}{L_{0}^{21}(\lambda)}=\frac{<p, q>_{m}+\sqrt{-F_{1}}}{<A q, q>_{0}} \lambda^{2 m+1}\left[1+O\left(\lambda^{-2}\right)\right], \\
& \left.h_{+}^{(2)} h_{-}^{(1)}\right|_{(m, \lambda)}=\left(\lambda^{2}-\gamma^{2}\right)^{m} \frac{L_{m}^{11}(\lambda)-\rho_{\lambda}}{L_{0}^{21}(\lambda)}=\frac{<p, q>_{m}-\sqrt{-F_{1}}}{<A q, q>_{0}} \lambda^{2 m+1}\left[1+O\left(\lambda^{-2}\right)\right] .
\end{aligned}
$$


Thus we obtain Eq. (3.28) by solving $h_{ \pm}^{(2)}$ and using Eq. (3.27).

From Eq. (3.20) we have

$$
\begin{aligned}
& h_{ \pm}^{(2)}(2 k, \lambda)=\left(\lambda c_{\lambda}^{ \pm}\right) \lambda^{-1} M_{\gamma}^{21}(2 k, \lambda)+M_{\gamma}^{22}(2 k, \lambda) \\
& \lambda h_{ \pm}^{(2)}(2 k+1, \lambda)=\left(\lambda c_{\lambda}^{ \pm}\right) M_{\gamma}^{21}(2 k+1, \lambda)+\lambda M_{\gamma}^{22}(2 k+1, \lambda) .
\end{aligned}
$$

By Lemma 3.3 and the discussion on $\lambda c_{\lambda}^{ \pm}$, two meromorphic functions (the Baker functions) $H^{(2)}(2 k, \mathfrak{p})$ and $H^{(2)}(2 k+1, \mathfrak{p})$ are defined on $\mathcal{R}$, respectively, with

$$
\begin{aligned}
& H^{(2)}\left(2 k, \mathfrak{p}\left(\lambda^{2}\right)\right)=h_{+}^{(2)}(2 k, \lambda), \quad H^{(2)}\left(2 k,(\tau \mathfrak{p})\left(\lambda^{2}\right)\right)=h_{-}^{(2)}(2 k, \lambda), \\
& H^{(2)}\left(2 k+1, \mathfrak{p}\left(\lambda^{2}\right)\right)=\lambda h_{+}^{(2)}(2 k+1, \lambda), \quad H^{(2)}\left(2 k+1,(\tau \mathfrak{p})\left(\lambda^{2}\right)\right)=\lambda h_{-}^{(2)}(2 k+1, \lambda) .
\end{aligned}
$$

Proposition $3.6 H^{(2)}(2 k, \mathfrak{p})$ and $H^{(2)}(2 k+1, \mathfrak{p})$ have the divisors respectively,

$$
\begin{aligned}
& \Sigma_{j=1}^{g}\left[\mathfrak{p}\left(\nu_{j}^{2}(2 k)\right)-\mathfrak{p}\left(\nu_{j}^{2}(0)\right)\right]+2 k \mathfrak{p}\left(\gamma^{2}\right)-k\left(\infty_{+}+\infty_{-}\right), \\
& \Sigma_{j=1}^{g}\left[\mathfrak{p}\left(\nu_{j}^{2}(2 k+1)\right)-\mathfrak{p}\left(\nu_{j}^{2}(0)\right)\right]+(2 k+1) \mathfrak{p}\left(\gamma^{2}\right)+\mathfrak{o}-(k+1)\left(\infty_{+}+\infty_{-}\right) .
\end{aligned}
$$

Proof. From Eq. (3.26) and (3.29) we obtain

$$
\begin{aligned}
& H^{(2)}(2 k, \mathfrak{p}) H^{(2)}(2 k, \tau \mathfrak{p})=\frac{<A q, q>_{2 k}}{<A q, q>_{0}}\left(\zeta-\gamma^{2}\right)^{2 k} \prod_{j=1}^{g} \frac{\zeta-\nu_{j}^{2}(2 k)}{\zeta-\nu_{j}^{2}(0)} \\
& H^{(2)}(2 k+1, \mathfrak{p}) H^{(2)}(2 k+1, \tau \mathfrak{p})=\frac{<A q, q>_{2 k+1}}{<A q, q>_{0}} \zeta\left(\zeta-\gamma^{2}\right)^{2 k+1} \prod_{j=1}^{g} \frac{\zeta-\nu_{j}^{2}(2 k+1)}{\zeta-\nu_{j}^{2}(0)}
\end{aligned}
$$

where $\mathfrak{p}=\mathfrak{p}(\zeta)$. As $\mathfrak{p} \rightarrow \infty_{ \pm}$, by Eq. (3.28) and (3.29) we have

$$
\begin{aligned}
& H^{(2)}(2 k, \mathfrak{p})=\frac{<p, q>_{2 k} \mp \sqrt{-F_{1}}}{<p, q>_{0} \mp \sqrt{-F_{1}}} \zeta^{k}\left[1+O\left(\zeta^{-1}\right)\right] \\
& H^{(2)}(2 k+1, \mathfrak{p})=\frac{<p, q>_{2 k+1} \mp \sqrt{-F_{1}}}{<p, q>_{0} \mp \sqrt{-F_{1}}} \zeta^{k+1}\left[1+O\left(\zeta^{-1}\right)\right] .
\end{aligned}
$$

By these formulas it is easy to calculate the divisors.

By using the technique developed by Toda [28], based on the meromorphic differentials $\mathrm{d} \ln H^{(2)}(2 k, \mathfrak{p})$ and $\mathrm{d} \ln H^{(2)}(2 k+1, \mathfrak{p})$, immediately we get

$$
\begin{aligned}
& \sum_{j=1}^{g} \int_{\mathfrak{p}\left(\nu_{j}^{2}(0)\right)}^{\mathfrak{p}\left(\nu_{j}^{2}(2 k)\right)} \vec{\omega}+k\left(\int_{\infty_{+}}^{\mathfrak{p}\left(\gamma^{2}\right)} \vec{\omega}+\int_{\infty_{-}}^{\mathfrak{p}\left(\gamma^{2}\right)} \vec{\omega}\right) \equiv 0, \quad(\bmod \mathcal{T}), \\
& \sum_{j=1}^{g} \int_{\mathfrak{p}\left(\nu_{j}^{2}(0)\right)}^{\mathfrak{p}\left(\nu_{j}^{2}(2 k+1)\right)} \vec{\omega}+(k+1)\left(\int_{\infty_{+}}^{\mathfrak{p}\left(\gamma^{2}\right)} \vec{\omega}+\int_{\infty_{-}}^{\mathfrak{p}\left(\gamma^{2}\right)} \vec{\omega}\right)+\int_{\mathfrak{p}\left(\gamma^{2}\right)}^{\mathfrak{o}} \vec{\omega} \equiv 0, \quad(\bmod \mathcal{T}),
\end{aligned}
$$


where $\vec{\omega}=\left(\omega_{1}, \cdots, \omega_{g}\right)^{T}$ are the normalized basis of holomorphic differentials on $\mathcal{R}$, while $\mathcal{T}$ is the basic lattice spanned by the periodic vectors of $\mathcal{R}$ [8, 11]. With the help of the Abel map $\mathcal{A}: \operatorname{Div}(\mathcal{R}) \rightarrow J(\mathcal{R}), \mathcal{A}(\mathfrak{p})=\int_{\mathfrak{p}_{0}}^{\mathfrak{p}} \vec{\omega}$, the Abel-Jacobi variable is defined as

$$
\phi(m)=\mathcal{A}\left(\sum_{j=1}^{g} \mathfrak{p}\left(\nu_{j}^{2}(m)\right)\right) .
$$

This endows Eq. (3.33) with a clear geometric explanation.

Proposition 3.7 In the Jacobi variety $J(\mathcal{R})=\mathbb{C}^{g} / \mathcal{T}$, the discrete flow $\mathcal{S}_{\gamma}^{m}$ is linearized by the Abel-Jacobi variable

$$
\phi(m) \equiv \phi(0)+m \Omega_{\gamma}+\delta_{m} \Omega_{0 \gamma}, \quad(\bmod \mathcal{T})
$$

where $\delta_{2 k}=0, \delta_{2 k+1}=1$, and

$$
\Omega_{\gamma}=\frac{1}{2}\left(\int_{\mathfrak{p}\left(\gamma^{2}\right)}^{\infty+} \vec{\omega}+\int_{\mathfrak{p}\left(\gamma^{2}\right)}^{\infty} \vec{\omega}\right), \quad \Omega_{0 \gamma}=\Omega_{\gamma}+\int_{\mathfrak{o}}^{\mathfrak{p}\left(\gamma^{2}\right)} \vec{\omega} .
$$

The meromorphic function $H^{(2)}(2 k, \mathfrak{p})$ is expressed by its divisor up to a constant factor

$$
\begin{aligned}
H^{(2)}(2 k, \mathfrak{p})= & \text { const } \cdot \frac{\theta[-\mathcal{A}(\mathfrak{p})+\phi(2 k)+K]}{\theta[-\mathcal{A}(\mathfrak{p})+\phi(0)+K]} \\
& \cdot \exp \left\{k \int_{\mathfrak{p}_{0}}^{\mathfrak{p}} \omega\left[\mathfrak{p}\left(\gamma^{2}\right), \infty_{+}\right]+\omega\left[\mathfrak{p}\left(\gamma^{2}\right), \infty_{-}\right]\right\},
\end{aligned}
$$

where $K$ is the Riemann constant vector and $\omega[\mathfrak{p}, \mathfrak{q}]$ is an Abel differential of the third kind, possessing only two simple poles at $\mathfrak{p}, \mathfrak{q}$ with residues $+1,-1$, respectively. Resorting to Eq. (3.32), by the asymptotic behaviors of Eq. (3.37) near $\infty_{ \pm}$we obtain

$$
\begin{aligned}
& \frac{u_{2 k}-\sqrt{-F_{1}}}{u_{0}-\sqrt{-F_{1}}}=\text { const } \cdot \frac{\theta\left[-\mathcal{A}\left(\infty_{+}\right)+\phi(2 k)+K\right]}{\theta\left[-\mathcal{A}\left(\infty_{+}\right)+\phi(0)+K\right]}\left(r_{\gamma}^{+} r_{\gamma,-}^{+}\right)^{k}, \\
& \frac{u_{2 k}+\sqrt{-F_{1}}}{u_{0}+\sqrt{-F_{1}}}=\mathrm{const} \cdot \frac{\theta\left[-\mathcal{A}\left(\infty_{-}\right)+\phi(2 k)+K\right]}{\theta\left[-\mathcal{A}\left(\infty_{-}\right)+\phi(0)+K\right]}\left(r_{\gamma}^{-} r_{\gamma,+}^{-}\right)^{k},
\end{aligned}
$$

where

$$
r_{\gamma}^{ \pm}=\lim _{\mathfrak{p} \rightarrow \infty_{ \pm}} \frac{1}{\zeta(\mathfrak{p})} \exp \int_{\mathfrak{p}_{0}}^{\mathfrak{p}} \omega\left[\mathfrak{p}\left(\gamma^{2}\right), \infty_{ \pm}\right], \quad r_{\gamma, \mp}^{ \pm}=\exp \int_{\mathfrak{p}_{0}}^{\infty_{ \pm}} \omega\left[\mathfrak{p}\left(\gamma^{2}\right), \infty_{\mp}\right]
$$

We introduce a new variable $v_{m}$ by

$$
v_{m}=\frac{u_{m}-\sqrt{-F_{1}}}{u_{m}+\sqrt{-F_{1}}}, \quad u_{m}=\sqrt{-F_{1}} \frac{1+v_{m}}{1-v_{m}} .
$$


Cancelling the constant factor in Eq. (3.38), we arrive at

$$
v_{2 k}=v_{0} \cdot \frac{\theta\left[2 k \Omega_{\gamma}+\Omega+K(0)\right] \cdot \theta[K(0)]}{\theta\left[2 k \Omega_{\gamma}+K(0)\right] \cdot \theta[\Omega+K(0)]} \cdot e^{2 k R_{\gamma}},
$$

where $\Omega=\int_{\infty_{+}}^{\infty_{-}} \vec{\omega}$ and

$$
\begin{aligned}
& -\mathcal{A}\left(\infty_{-}\right)=\int_{\infty_{-}}^{\mathfrak{p}_{0}} \vec{\omega}=\eta_{-}, \quad-\mathcal{A}\left(\infty_{+}\right)=\Omega+\eta_{-}, \\
& K(m)=\phi(m)+K+\eta_{-}, \quad R_{\gamma}=\frac{1}{2} \ln \left[\left(r_{\gamma}^{+} r_{\gamma,-}^{+}\right) /\left(r_{\gamma}^{-} r_{\gamma,+}^{-}\right)\right]
\end{aligned}
$$

Similarly, considering the analytic expression for $H^{(2)}(2 k+1, \mathfrak{p})$ leads to

$$
v_{2 k+1}=v_{0} \cdot \frac{\theta\left[(2 k+1) \Omega_{\gamma}+\Omega_{0 \gamma}+\Omega+K(0)\right] \cdot \theta[K(0)]}{\theta\left[(2 k+1) \Omega_{\gamma}+\Omega_{0 \gamma}+K(0)\right] \cdot \theta[\Omega+K(0)]} \cdot e^{(2 k+1) R_{\gamma}+R_{0 \gamma}},
$$

where

$$
R_{0 \gamma}=R_{\gamma}+\ln r_{0 \gamma}, \quad r_{0 \gamma}=\exp \int_{\infty_{-}}^{\infty_{+}} \omega\left[\mathfrak{o}, \mathfrak{p}\left(\gamma^{2}\right)\right]
$$

Proposition 3.8 The finite genus potential $v_{m}$, defined by Eq. (3.11) and (3.40), has an explicit evolution formula along the discrete flow $\mathcal{S}_{\gamma}^{m}$,

$$
v_{m}=v_{0} \cdot \frac{\theta\left[m \Omega_{\gamma}+\delta_{m} \Omega_{0 \gamma}+K(0)+\Omega\right] \cdot \theta[K(0)]}{\theta\left[m \Omega_{\gamma}+\delta_{m} \Omega_{0 \gamma}+K(0)\right] \cdot \theta[K(0)+\Omega]} \cdot e^{m R_{\gamma}+\delta_{m} R_{0 \gamma}},
$$

where the vectors $K(m), \Omega_{\gamma}, \Omega_{0 \gamma}$ and $\Omega$ are given by Eq. (3.36) and (3.42), while the constants $R_{\gamma}, R_{0 \gamma}$ are defined by Eq. 3.42) and (3.44); moreover, $\delta_{2 k}=0, \delta_{2 k+1}=1$, for all $k$.

\section{Solutions of ISKdV equation (1.1)}

Let $\gamma_{1}, \gamma_{2}$ be the two constants given in Eq. (1.1). By proposition 3.2, setting $\gamma=\gamma_{1}, \gamma_{2}$ in the above we have two symplectic maps $\mathcal{S}_{\gamma_{1}}$ and $\mathcal{S}_{\gamma_{2}}$, sharing the same set of integrals $\left\{F_{l}\right\}$. Resorting to the discrete version of Liouville-Arnold theorem [25, 27, 29], they commute. Thus we have well-defined functions with two discrete arguments $m$ and $n$,

$$
\begin{aligned}
& (p(m, n), q(m, n))=\mathcal{S}_{\gamma_{1}}^{m} \mathcal{S}_{\gamma_{2}}^{n}\left(p_{0}, q_{0}\right), \\
& b_{m n}=f_{\gamma}(p(m, n), q(m, n)), \\
& u_{m n}=f_{U}(p(m, n), q(m, n))=<p(m, n), q(m, n)>, \\
& v_{m n}=\left(u_{m n}-\sqrt{-F_{1}}\right) /\left(u_{m n}+\sqrt{-F_{1}}\right) .
\end{aligned}
$$

Proposition 4.1 Both the functions $u_{m n}$ and $v_{m n}$, defined by Eq. [4.1), solve Eq. (1.1). 
Proof. By the commutativity of $\mathcal{S}_{\gamma_{1}}^{m}$ and $\mathcal{S}_{\gamma_{2}}^{n}$, we have

$$
(p(m, n), q(m, n))=\mathcal{S}_{\gamma_{1}}^{m}(p(0, n), q(0, n))=\mathcal{S}_{\gamma_{2}}^{n}(p(m, 0), q(m, 0))
$$

From Eq. (3.12) we obtain

$$
b_{m n}=\gamma_{1} /(u-\tilde{u})=\gamma_{2} /(u-\hat{u})
$$

By Eq. (3.6),$\chi_{j}=\left(p_{j}(m, n), q_{j}(m, n)\right)^{T}$ solves simultaneously

$$
\begin{array}{ll}
\tilde{\chi}_{j}=\left(\alpha_{j}^{2}-\gamma_{1}^{2}\right)^{-1 / 2} D^{\left(\gamma_{1}\right)}\left(\alpha_{j}, b_{m n}\right) \chi_{j}, & b_{m n}=\gamma_{1} /(u-\tilde{u}), \\
\hat{\chi}_{j}=\left(\alpha_{j}^{2}-\gamma_{2}^{2}\right)^{-1 / 2} D^{\left(\gamma_{2}\right)}\left(\alpha_{j}, b_{m n}\right) \chi_{j}, & b_{m n}=\gamma_{2} /(u-\hat{u}) .
\end{array}
$$

Thus $u_{m n}$ satisfies Eq. (1.1) by Eq. (1.11). In order to prove that $v_{m n}$ is also a solution, it is sufficient to notice that $(i) F_{1}$ is a constant of motion which is independent of $m$ and $n$; (ii) Eq. (1.1) is invariant under the Möbius transformation $u \mapsto v$ given by Eq. (4.1).

Apply Eq. (3.45) to the flow $\mathcal{S}_{\gamma_{1}}^{m}$ and $\mathcal{S}_{\gamma_{2}}^{n}$ successively. By $v_{00} \rightarrow v_{m 0} \rightarrow v_{m n}$ we obtain

Proposition 4.2 The $l S K d V$ equation (1.1) has finite genus solutions

$$
\begin{aligned}
& v_{m n}=v_{00} \cdot \frac{\theta\left[m \Omega_{\gamma_{1}}+n \Omega_{\gamma_{2}}+\delta_{m} \Omega_{0 \gamma_{1}}+\delta_{n} \Omega_{0 \gamma_{2}}+K_{00}+\Omega\right] \cdot \theta\left[K_{00}\right]}{\theta\left[m \Omega_{\gamma_{1}}+n \Omega_{\gamma_{2}}+\delta_{m} \Omega_{0 \gamma_{1}}+\delta_{n} \Omega_{0 \gamma_{2}}+K_{00}\right] \cdot \theta\left[K_{00}+\Omega\right]} \\
& \cdot \exp \left(m R_{\gamma_{1}}+n R_{\gamma_{2}}+\delta_{m} R_{0 \gamma_{1}}+\delta_{n} R_{0 \gamma_{2}}\right),
\end{aligned}
$$

and $u_{m n}=\sqrt{-F_{1}}\left(1+v_{m n}\right) /\left(1-v_{m n}\right)$. Further, any Möbius transformation $w_{m n}=$ $\left(a_{11} v_{m n}+a_{12}\right) /\left(a_{21} v_{m n}+a_{22}\right)$ solves Eq. (1.1), where $a_{j k}$ are constants.

Acknowledgment. This work is supported by National Natural Science Foundation of China (Grant Nos. 11426206; 11501521), State Scholarship Found of China (CSC No. 201907045035), and Graduate Student Education Research Foundation of Zhengzhou University (Grant No. YJSXWKC201913). We would like to thank Prof. Frank W Nijhoff and Prof. Da-jun Zhang for helpful discussions.

\section{References}

[1] V.E. Adler, A.I. Bobenko and Yu.B. Suris, Classification of integrable equations on quadgraphs, Comm. Math. Phys. 233 (2003) 513-543.

[2] V.I. Arnold, Mathematical Methods of Classical Mechanics (Springer, Berlin, 1978). 
[3] J. Atkinson, J. Hietarinta and F. Nijhoff, Seed and soliton solutions of Adlers lattice equation, J. Phys. A: Math. Theor. 40 (2006) F1-F8.

[4] M. Bruschi and O. Ragnisco, Nonlinear differential-difference equations, associated Bäcklund transformations and Lax technique, J. Phys. A: Math. Gen. 14 (1981) 1075-1081.

[5] C.W. Cao, A classical integrable system and the involutive representation of solutions of the KdV equations, Acta Math. Sinica: New Series 7 (1991) 216-223.

[6] C.W. Cao and X.X. Xu, A finite genus solution of the H1 model, J. Phys. A: Math. Gen. 45 (2012) 055213(13pp).

[7] C.W. Cao and G.Y. Zhang, Integrable symplectic maps associated with the ZS-AKNS spectral problem, J. Phys. A: Math. Theor. 45 (2012) 265201(15pp).

[8] H.M. Farkas and I. Kra, Riemann Surfaces (Springer, New York, 1992).

[9] F. Gesztesy, H. Holden, J. Michor and G. Teschl, Soliton Equations and Their Algebrogeometric Solutions. Volumn II: (1+1)-dimensional Discrete Models (Cambridge University Press, Cambridge, 2008).

[10] B. Grammaticos, Y. Kosmann-Schwarzbach and T. Tamizhmani, Discrete Integrable Systems (Springer, Berlin, 2004).

[11] P. Griffiths and J. Harris, Principles of Algebraic Geometry (Wiley, New York, 1978).

[12] J. Hietarinta, N. Joshi and F.W. Nijhoff, Discrete Systems and Integrablity (Cambridge University Press, Cambridge, 2016).

[13] A.N.W. Hone, V.B. Kuznetsov and O. Ragnisco, Bäcklund transformations for many body systems related to KdV, J. Phys. A: Math. Gen. 32(27) (1999) L299-L306.

[14] J. Hietarinta and C. Viallet, Weak Lax pairs for lattice equations, Nonlinearty 25 (2011) 1955-1966.

[15] J. Hietarinta and D.J. Zhang, Soliton solutions for ABS lattice equations: II. Casorations and bilinearization, J. Phys. A: Math. Theor. 42 (2009) 404006(30pp).

[16] V.B. Kuznetsov and P. Vanhaecke, Bäcklund transformations for finite dimensional integrable systems: A geometric approach, Journal of Geometry and Physics 44(1) (2000) 1-40.

[17] G. Lamé, Leçons sur les Coordonnées Curvilignes et leurs Diverses Applications (Mallet-Bachelier, Paris, 1859).

[18] D. Levi and R. Benguria, Bäcklund transformations and nonlinear differential difference equations, Proc. Natl. Acad. Sci. USA. 77 (1980) 5025-5027. 
[19] D. Levi, M. Petrera and C. Scimiterna, The lattice Schwarzian KdV equation and its symmetries, J. Phys. A: Math. Theor. 40 (2007) 12753-12761.

[20] S.Y. Lou, Conformal invariance and integrable models, J. Phys. A: Math. Gen. 30 (1997) 4803-4813.

[21] F.W. Nijhoff, J. Atkinson and J. Hietarinta, Soliton solutions for ABS lattice equation: I. Cauchy matrix approach, J. Phys. A: Math. Theor. 42 (2009) 404005(34pp).

[22] F.W. Nijhoff and J. Atkinson, Elliptic $N$-soliton solutions of ABS lattice equations, Int. Math. Res. Not. 2010 (2010) 3837-3895.

[23] F.W. Nijhoff and H.W. Capel, The discrete Korteweg-de Vries equation, Acta Appl. Math. 39 (1995) 133-158.

[24] F.W. Nijhoff, A.N.W. Hone and N. Joshi, On a Schwarzian PDE associated with the KdV hierarchy, Phys. Lett. A 267(2-3) (2000) 147-156.

[25] G.R.W. Quispel, J.A.G. Roberts and C.J. Thompsonb, Integrable mappings and soliton equations. Phys. Lett. A 126 (1988) 419-421.

[26] A.G. Rasin, Infinitely many symmetries and conservation laws for quad-graph equations via the Gardner method, J. Phys. A: Math. Theor. 43 (2010) 235201(11pp).

[27] Yu.B. Suris, The Problem of Integrable Discretization: Hamiltonian Approach (Birkhäuser, Basel, 2003).

[28] M. Toda, Theory of Nonlinear Lattices (Springer, Berlin, 1981).

[29] A P. Veselov, Integrable maps. Russ. Math. Surv. 46 (1991) 3-45.

[30] J. Weiss, M. Tabor and G. Carnevale, The Painléve property for partial differential equations, J. Math. Phys. 24 (1983) 522-526.

[31] J. Weiss, The Painléve property for partial differential equations. II: Bäcklund transformation, Lax pairs, and the Schwarzian derivative, J. Math. Phys. 24 (1983) 14051413.

[32] P. Xenitidis, Symmetries and conservation laws of the ABS equations and corresponding differential difference equations of Volterra type, J. Phys. A: Math. Theor. 44 (2011) 435201(22pp).

[33] D.D. Zhang and D.J. Zhang, On decomposition of the ABS lattice equations and related Bäcklund transformations, J. Nonl. Math. Phys. 25 (2018) 34-53. 